\title{
REGARDING STOPPING RULES FOR BROWNIAN MOTION AND RANDOM WALKS ${ }^{1}$
}

\author{
BY LEROY H. WALKER
}

Communicated by David Blackwell, July 15, 1968

At the Fifth Berkeley Symposium on Mathematical Statistics and Probability, A. Dvoretzky [4] presented a paper on "Certain Optimal Stopping Rules." In this paper he proved the existence of an optimal stopping rule for the following situation: let $X_{1}, X_{2}, X_{3}, \cdots$ be a sequence of independent, identically distributed, real-valued random variables with zero means and unit variances defined on the probability space $\left(\Omega_{1}, \digamma_{1}, P_{1}\right)$. Then for (fixed) $\beta>\frac{1}{2}$, there exists a positive, integer-valued random variable $n$ (called a stopping rule) defined on $\Omega_{1}$, whose value for any sample point $\omega$ is a function only of the observable variables $X_{1}(\omega), X_{2}(\omega), \cdots, X_{n(\omega)}(\omega)$ and which realizes the maximum value of $E\left\{n^{-\beta} \sum_{i=1}^{n} X_{i}\right\}$ over all such random variables, i.e. stopping rules. In his proof, Dvoretzky was lead to conjecture concerning the asymptotic behavior of the stopping boundary which provided the definition of his optimal rule. Theorem 1 of this announcement provides an explicit description of the asymptotic behavior of this boundary. Theorem 2 gives an optimal stopping rule for an analogous situation for a continuous time process, namely Brownian motion.

The paper of Y.S. Chow and H. Robbins [2], which initiated work in this area of stopping rule problems, and the paper of $\mathrm{H}$. Teicher and J. Wolfowitz [7] are also related to the results announced herein. Many of the ideas used to achieve these results were suggested by techniques exhibited in the paper by Teicher and Wolfowitz. While completing the writeup of the results announced herein, the work of L. A. Shepp [5] came to the attention of the author, in which Shepp obtained the same results as given by Theorem 1 and Theorem 2 for the case when $\beta=1$.

Concise statements of these two theorems require the definition of some notation. The sequence $X_{1}, X_{2}, X_{3}, \cdots$ is as suggested above,

\footnotetext{
1 Complete proofs of all statements made in this announcement can be found in the author's Ph.D. dissertation, Stopping rules for Brownian motion and random walks, University of California at Los Angeles, 1968, which was written under the direction of Professor C. J. Stone and was supported in part by the National Science Foundation through grant GP-5224. Some generalizations of the results presented in this announcement are also contained in the dissertation.
} 
and $C_{1}, C_{2}, C_{3}, \cdots$ is a sequence of positive real numbers. Define $N$ to be the collection of all stopping rules, i.e. functions $n: \Omega_{1} \rightarrow I_{+} \cup\{\infty\}$, where $I_{+}$denotes the set of positive integers, with the properties that for $k \in I_{+}$the event $\{n \leqq k\}$ belongs to $\mathscr{F}_{1}\left(X_{1}, \cdots, X_{k}\right)$, where $F_{1}\left(X_{1}, \cdots, X_{k}\right)$ denotes the minimum $\sigma$-algebra of elements of $F_{1}$ in which $X_{1}, X_{2}, \cdots, X_{k}$ are all measurable functions, and $P_{1}(\{n<\infty\})=1$. Dvoretzky's results, when $C_{k}=k^{-\beta}$ with $\beta>\frac{1}{2}$, were that $\sup _{n \in N} E\left\{C_{n} \sum_{i=1}^{n} X_{i}\right\}$ is finite and that there exists a sequence $b_{1}, b_{2}, b_{3}, \cdots$ of positive real numbers with the property that the function $n^{\prime}: \Omega_{1} \rightarrow I_{+} \cup\{\infty\}$ defined by

$$
n^{\prime}(\omega)=\sup \left\{k: \sum_{i=1}^{j} X_{i}(\omega)<b_{j} \text { for } j=1,2, \cdots, k-1\right\}
$$

belongs to $N$ and satisfies the equation

$$
E\left\{C_{n^{\prime}} \sum_{i=1}^{n^{\prime}} X_{i}\right\}=\sup _{n \in N} E\left\{C_{n} \sum_{i=1}^{n} X_{i}\right\} .
$$

Such an $n^{\prime}$ is said to be an optimal stopping rule. Dvoretzky's question was, "Does the limit of $b_{m} /(m)^{1 / 2}$ as $m$ tends to infinity exist?"

ThEOREM 1. The limit of $b_{m} /(m)^{1 / 2}$ exists and is equal to $y_{0}$ where $y_{0}$ is the unique positive real root of the equation

$$
\int_{0}^{\infty} t^{2(\beta-1)} e^{\left(y t-t^{2} / 2\right)} d t=y \int_{0}^{\infty} t^{(2 \beta-1)} e^{\left(y t-t^{2} / 2\right)} d t .
$$

The proof of this theorem is long and technically involved. One key idea in the proof is that the $b$ 's are determined by the relation

$$
\sup _{n \in N} E\left\{C_{m+n}\left(y+\sum_{i=1}^{n} X_{i}\right)-C_{n} y\right\} \begin{cases}>0 & \text { when } y<b_{m} \\ \leqq 0 & \text { when } y \geqq b_{m} .\end{cases}
$$

This relation was used by Teicher and Wolfowitz in their work on stopping rules in this situation.

As the first step in the proof, a continuous analog to this discrete stopping rule situation is considered; namely, let $W$ be a real-valued Brownian motion with mean zero and variance parameter one defined on the probability space $\left(\Omega_{2}, F_{2}, P_{2}\right)$. Let $C$ denote a function defined by $C(t)=\alpha^{t}$ with $0<\alpha<1$ or $C(t)=(1+t)^{-\beta}$ with $\beta>\frac{1}{2}$ for $t \geqq 0$. Define $T$ as the collection of all functions $\tau: \Omega_{2} \rightarrow R_{+} \cup\{\infty\}$, where $R_{+}$denotes the nonnegative real line, with the properties that for any $r \in R_{+}$the event $\{\tau \leqq r\}$ belongs to $\mathcal{F}_{2}\left(W_{\varepsilon}: s \leqq r\right)$, where $F_{2}\left(W_{8}: s \leqq r\right)$ denotes the minimum $\sigma$-algebra of elements of $F_{2}$ in

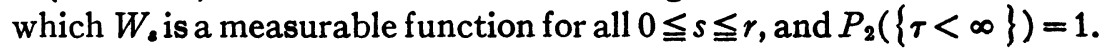


THEOREM 2. $\sup _{r \in T} E\left\{C(\tau) W_{r}\right\}$ is finite and there exists a function $f: R_{+} \rightarrow R_{+}$determined by the relation

$$
\sup _{r \in \boldsymbol{T}} E\left\{C(r+\tau)\left(y+W_{\tau}\right)-C(r) y\right\} \begin{cases}>0 & \text { when } y<f(r) \\ \leqq 0 & \text { when } y \geqq f(r)\end{cases}
$$

such that the function $\tau^{\prime}: \Omega_{1} \rightarrow R_{+} \cup\{\infty\}$ defined by

$$
\tau^{\prime}(\omega)=\sup \left\{t: W_{s}<f(s) \text { for } 0 \leqq s<t\right\}
$$

is an optimal stopping rule, i.e. $\tau^{\prime}$ belongs to $T$ and

$$
E\left\{C\left(\tau^{\prime}\right) W_{\tau^{\prime}}\right\}=\sup _{\tau \in T} E\left\{C(\tau) W_{\tau}\right\} .
$$

When $C(t)=\alpha^{t}, f(r)=\left((2 \log (1 / \alpha))^{1 / 2}\right)^{-1}$.

When $C(t)=(1+t)^{-\beta}, f(r)=y_{0}(1+r)^{1 / 2}$ where $y_{0}$ is the same value as given above for the limit of $b_{m} /(m)^{1 / 2}$.

The proof that $\tau^{\prime}$ is optimal is accomplished by restricting analysis to stopping rules which belong to a sequence of subsets of $T$. The subsets are selected such that the range spaces of the considered stopping rules are well-ordered, discrete, and become dense in $R_{+}$as the subset index tends to infinity. The former two properties permit proofs of optimality over the set of restricted stopping rules because a given rule can be modified on a subset of $\Omega_{2}$ conditioned on stopping at a particular time. Since such modifications are required at most a countable number of times, measurability properties of the resulting rules remain intact. The denseness property insures that the optimal stopping rules over the subsets of $T$ converge to $\tau^{\prime}$, thereby establishing the optimality of $\tau^{\prime}$. This optimality proof depends only on properties following from the relation determining $f(r)$. The explicit value of $f(r)$ is obtained by using D. A. Darling and A. J. F. Siegert's [3] results concerning the first contact time of a continuous Markov process with a constant boundary $y$ which gives a formula for $E\left\{C(\tau) W_{\tau}\right\}$ as a function of $y$. The value of $y$ which makes this expression maximum then determines $f(r)$ explicitly.

As the next step in the proof of the result pertaining to the limit of $b_{m} /(m)^{1 / 2}$, the relation determining the $b$ 's is expressed in terms of a sampled Brownian motion using Skorokhod's representation of sums of independent, identically distributed random variables. ${ }^{2}$ This representation in the form given by $\mathrm{L}$. Breiman ${ }^{3}$ provides the existence of

${ }^{2}$ See the theorem on p. 180 of [6] (p. 163 in the English translation).

${ }^{3}$ Theorem 13.6, p. 276, of [1]. 
a sequence $\bar{X}_{1}, \bar{X}_{2}, \bar{X}_{3}, \cdots$ of independent, identically distributed, pairs of real-valued random variables defined on a probability space $\left(\bar{\Omega}_{1}, \bar{F}_{1}, \bar{P}_{1}\right)$ such that a sequence $t(1), t(2), t(3), \cdots$ of nonnegative, independent, identically distributed random variables (called sample times) defined on $\left(\Omega_{2} \times \bar{\Omega}_{1}, F_{2} \times \bar{F}_{1}, P_{2} \times \bar{P}_{1}\right)$ can be constructed with the property that $X_{1}, X_{1}+X_{2}, X_{1}+X_{2}+X_{3}, \cdots$ and $W_{t(1)}$, $W_{t(1)+t(2)}, W_{t(1)+t(2)+t(3)}, \cdots$ have the same joint distribution functions. Then the determining relation for the $b$ 's is

$$
\begin{aligned}
\sup _{n \in N_{m}} E\left\{C\left(\frac{k+n}{m}\right)\left(y+W_{\nu(n, m)}\right)-C\left(\frac{k}{m}\right) y\right\} & \begin{cases}>0 & \text { when } y<b_{m} /(m)^{1 / 2} \\
\leqq 0 & \text { when } y \geqq b_{m} /(m)^{1 / 2}\end{cases}
\end{aligned}
$$

where $N_{m}$ and $\nu(n, m)$ are defined as follows. For $j \in I_{+}, m \nu(j, m)$ is the sum of the first $j$ sample times given by the Skorokhod representation theorem when the Brownian motion $W^{(m)}$, which is defined by $W_{i}^{(m)}=(m)^{1 / 2} W_{t / m}$, is used. For the definition of $N_{m}$, the concept of the past of the process up to time $\nu(j, m)$ is needed. Technically this concept can be expressed as: let $\mathcal{F}_{3}\left(W_{s}: s \leqq \nu(j, m)\right)$ denote the collection of all subsets $A$ of $\Omega_{2} \times \bar{\Omega}_{1}$ such that for every $r \in R_{+}$,

$$
A \cap\{\nu(j, m) \leqq r\} \in F_{3}\left(\bar{X}_{1}, \cdots, \bar{X}_{j} ; W_{s}: s \leqq r\right)
$$

where $\digamma_{3}\left(\bar{X}_{1}, \cdots, \bar{X}_{j} ; W_{:}: s \leqq r\right)$ is the minimum $\sigma$-algebra of elements of $F_{2} \times \bar{F}_{1}$ in which $\bar{X}_{1}, \bar{X}_{2}, \cdots, \bar{X}_{j}$ and $W$, for $0 \leqq s \leqq r$ are all measurable functions. Then $N_{m}$ is the collection of all functions $n: \Omega_{2} \times \bar{\Omega}_{1} \rightarrow I_{+} \cup\{\infty\}$ with the properties that for every $k \in I_{+}$the event $\{n \leqq k\}$ belongs to $\mathcal{F}_{3}\left(W_{s}: s \leqq \nu(k, m)\right)$ and $P_{2} \times \bar{P}_{1}(\{n<\infty\})=1$. Proof of this form of the relation determining the $b$ 's involves proving the existence of an optimal stopping rule of the form

$$
n^{\prime}(\omega)=\sup \left\{k: y+W_{\nu(j, m)}<g_{m}(j) \text { for } j=1,2, \cdots, k-1\right\}
$$

where the $g_{m}: I_{+} \rightarrow R_{+}$is defined by

$$
\sup _{n \in N_{m}} E\left\{C\left(\frac{j+n}{m}\right)\left(z+W_{\nu(n, m)}\right)-C\left(\frac{j}{m}\right) z\right\}
$$

$$
\begin{cases}>0 & \text { when } z<g_{m}(j) \\ \leqq 0 & \text { when } z \geqq g_{m}(j) .\end{cases}
$$

Optimal in this case means $n^{\prime} \in N_{m}$ and

$$
E\left\{C\left(\frac{n^{\prime}}{m}\right)\left(y+W_{\nu\left(n^{\prime}, m\right)}\right)\right\}=\sup _{n \in N_{m}} E\left\{C\left(\frac{n}{m}\right)\left(y+W_{\nu(n, m)}\right)\right\} .
$$


As the last step in the proof of the result pertaining to the limit of $b_{m} /(m)^{1 / 2}$, the limit of $\sup _{n \in N_{m}} E\left\{C(n / m)\left(y+W_{\nu(n, m)}\right)-y\right\}$ as $m$ tends to infinity is shown to exist and to equal $\sup _{\tau \in T} E\left\{C(\tau)\left(y+W_{\tau}\right)-y\right\}$. This result then provides a proof of $\lim _{\inf _{m \rightarrow \infty}} b_{m} /(m)^{1 / 2} \geqq y_{0}$ by assuming that lim $\inf _{m \rightarrow \infty} b_{m} /(m)^{1 / 2}<y_{0}$, which leads directly to a contradiction. The result that $\lim \sup _{m \rightarrow \infty} b_{m} /(m)^{1 / 2} \leqq y_{0}$ is not as immediate. For this result, the procedure used is to restrict analysis to subsets of $N_{m}$ and $T$ for which the above limit result still holds and the supremum of $E\left\{C(\tau)\left(y+W_{\tau}\right)-y\right\}$ over the subset of $T$ is a strictly decreasing function of $y$. This property, coupled with the properties of the sequence $g_{m}(1), g_{m}(2), g_{m}(3), \cdots$ used to define the optimal rule $n^{\prime}$ described in the last paragraph, then leads to a proof of $\lim \sup _{m \rightarrow \infty} b_{m} /(m)^{1 / 2} \leqq y_{0}$.

\section{REFERENCES}

1. L. Breiman, Probability, Addison-Wesley, Reading, Mass., 1968.

2. Y. S. Chow and H. Robbins, On optimal stopping rules for $s_{n} / n$, Illinois J. Math. 9 (1965), 444-454.

3. D. A. Darling and A. J. F. Siegert, The first passage problem for a continuous Markov process, Ann. Math. Statist. 24 (1953), 624-639.

4. A. Dvoretzky, Existence and properties of certain optimal stopping rules, Proc. Fifth Berkeley Sympos. Math. Statis. Prob., University Press, Berkeley, Calif., vol. 1, 1967, pp. 441-452.

5. L. A. Shepp, Explicit solutions to some problems of optimal stopping, Ann. Math. Statist. (to appear).

6. A. V. Skorokhod, Studies in the theory of random processes, University of Kiev, Kiev (1961); English transl., Addison-Wesley, Reading, Mass., 1965.

7. $\mathrm{H}$. Teicher and J. Wolfowitz, Existence of optimal stopping rules for linear and quadratic rewards, Z. Wahrscheinlichkeitstheorie und Verw. Gebiete, 5 (1966), 361368.

University of California, los Angeles, California 90024 